\title{
A POTENTIAL GALAXY THRESHING SYSTEM IN THE COSMOS FIELD ${ }^{1}$
}

\author{
S. S. Sasaki, ${ }^{2,3}$ Y. Taniguchi ${ }^{3}$ N. Scoville, ${ }^{4,5}$ B. Mobasher,${ }^{6}$ H. Aussel, ${ }^{7}$ D. B. Sanders, ${ }^{8}$ A. Koekemoer, ${ }^{6}$ \\ M. Ajiki, ${ }^{2}$ Y. Komiyama, ${ }^{9}$ S. Miyazaki, ${ }^{10}$ N. Kaifu, ${ }^{9}$ H. Karoji, ${ }^{10}$ S. Okamura, ${ }^{11}$ N. Arimoto, ${ }^{9}$ \\ K. Ohta, ${ }^{12}$ Y. Shioya, ${ }^{3}$ T. Murayama, ${ }^{2}$ T. Nagao, ${ }^{9,13}$ J. Koda, ${ }^{4}$ L. Hainline, ${ }^{4}$ A. Renzini, ${ }^{14}$ \\ M. Giavalisco, ${ }^{6}$ O. Le Fèvre, ${ }^{15}$ C. Impey, ${ }^{16}$ M. Elvis, ${ }^{17}$ S. Lilly, ${ }^{18}$ \\ M. Rich, ${ }^{19}$ E. SchinNerer, ${ }^{20}$ AND K. Sheth ${ }^{4,21}$ \\ Received 2006 April 24; accepted 2006 November 5
}

\begin{abstract}
We report on the discovery of a new potential galaxy threshing system in the COSMOS 2 square degree field using the prime-focus camera, Suprime-Cam, on the $8.2 \mathrm{~m}$ Subaru Telescope. This system consists of a giant elliptical galaxy with $M_{V} \approx-21.6$ and a tidally disrupted satellite galaxy with $M_{V} \approx-17.7$ at a photometric redshift of $z \approx 0.08$. This redshift is consistent with the spectroscopic redshift of 0.079 for the giant elliptical galaxy obtained from the Sloan Digital Sky Survey (SDSS) archive. The luminosity masses of the two galaxies are $3.7 \times 10^{12}$ and $3.1 \times 10^{9} \mathcal{M}_{\odot}$, respectively. The distance between the two galaxies is greater than $100 \mathrm{kpc}$. The two tidal tails emanating from the satellite galaxy extend over $150 \mathrm{kpc}$. This system would be the second well-defined galaxy threshing system found so far. Subject headings: galaxies: evolution — galaxies: interactions
\end{abstract}

\section{INTRODUCTION}

Hierarchical clustering scenarios suggest that present-day galaxies assembled from much smaller building blocks during the course of their evolution (e.g., Peebles 1993). Recent deep surveys have found small dwarf galaxies (i.e., building blocks) at $z>2$

${ }^{1}$ Based on data collected at Subaru Telescope, which is operated by the National Astronomical Observatory of Japan.

${ }_{2}$ Astronomical Institute, Graduate School of Science, Tohoku University, Aramaki, Aoba, Sendai 980-8578, Japan.

${ }^{3}$ Physics Department, Graduate School of Science and Engineering, Ehime University, 2-5 Bunkyo-cho, Matsuyama 790-8577, Japan.

${ }^{4}$ California Institute of Technology, MC 105-24, 1200 East California Boulevard, Pasadena, CA 91125.

5 Visiting Astronomer, University of Hawaii, 2680 Woodlawn Drive, Honolulu, HI 96822.

${ }^{6}$ Space Telescope Science Institute, 3700 San Martin Drive, Baltimore, MD 21218.

7 Service d'Astrophysique, CEA/Saclay, 91191 Gif-sur-Yvette, France.

${ }^{8}$ Institute for Astronomy, 2680 Woodlawn Drive, University of Hawaii, Honolulu, HI 96822.

9 National Astronomical Observatory of Japan, 2-21-1 Osawa, Mitaka, Tokyo 181-8588, Japan.

${ }^{10}$ Subaru Telescope, National Astronomical Observatory, 650 North A'ohoku Place, Hilo, HI 96720

${ }^{11}$ Department of Astronomy, Graduate School of Science, University of Tokyo, 7-3-1 Hongo, Bunkyo-ku, Tokyo 113-0033, Japan.

12 Department of Astronomy, Graduate School of Science, Kyoto University, Kitashirakawa, Sakyo-ku, Kyoto 606-8502, Japan

${ }_{13}$ INAF-Osservatorio Astrofisico di Arcetri, Largo Enrico Fermi 5, 50125 Firenze, Italy.

${ }^{14}$ European Southern Observatory, Karl-Schwarzschild-Strasse 2, D-85748 Garching, Germany.

${ }_{15}$ Laboratoire d'Astrophysique de Marseille, BP 8, Traverse du Siphon, 13376 Marseille Cedex 12, France.

${ }^{16}$ Steward Observatory, University of Arizona, 933 North Cherry Avenue, Tucson, AZ 85721.

${ }^{17}$ Harvard-Smithsonian Center for Astrophysics, 60 Garden Street, Cambridge, MA 02138.

${ }_{18}$ Department of Physics, Swiss Federal Institute of Technology (ETH-Zurich), CH-8093 Zurich, Switzerland.

${ }^{19}$ Department of Physics and Astronomy, University of California, Los Angeles, CA 90095.

20 Max Planck Institut für Astronomie, Königstuhl 17, Heidelberg D-69117, Germany.

${ }_{21}$ Spitzer Science Center, California Institute of Technology, Pasadena, CA 91125. (e.g., Pascarelle et al. 1998; Ellis et al. 2001; Taniguchi et al. 2003a; Santos et al. 2004; Kneib et al. 2004; for a review see Taniguchi et al. 2003b). At the present epoch, the assembly process continues with large galaxies capturing their satellite galaxies. In order to understand the mass assembly of galaxies, it is therefore important to investigate such minor merger processes in detail.

It is known that most galaxies in the local universe have satellite galaxies (e.g., Zaritsky et al. 1997 and references therein). Satellite galaxies are expected to sink to the center of the potential of their host galaxies due to dynamical friction (Ostriker \& Tremaine 1975; Tremaine 1981); however, satellites with long dynamical friction timescales are still found orbiting their hosts. The satellites suffer from tidal disruption as they orbit in the galaxy's host dark matter halo. Therefore, the kinematic and morphological properties of the satellites can be used to investigate the structure and potential of the host dark matter halo.

The recent discovery of ultra compact dwarf (UCD) galaxies in the Fornax cluster suggests that such tidal disruption processes can yield small satellite galaxies (Drinkwater et al. 2000a, 2000b; Bekki et al. 2001, 2003). As dwarf galaxies orbit around the host galaxy, their outer stellar components are tidally removed. This process is also referred to as galaxy threshing. Recently, Forbes et al. (2003) identified the first case of a galaxy threshing system in an early release image of the Advanced Camera for Surveys (ACS) on the Hubble Space Telescope (Tran et al. 2003; de Grijp et al. 2003). The ACS image clearly shows faint, long tidal tails emanating from a satellite galaxy around an edge-on spiral galaxy at $z=0.145$.

Although a pair of tidal tails is often found in major mergers (Arp 1966; Toomre \& Toomre 1972), it is rare to find such tails in ongoing minor mergers. In this respect, the discovery of Forbes et al. (2003) provides important information of tidal disruption of satellite galaxies around the host galaxy. Following Bekki et al. (2001) we use the term "galaxy threshing" for such ongoing, tidally disrupted satellite galaxies.

Here, we present the serendipitous discovery of a new potential galaxy threshing system found in the COSMOS 2 square degree field (Scoville et al. 2006). The most important point is that we find clear evidence for the disruption of a small satellite galaxy. Destroyed systems are often observed, but faint tidal remnants are 

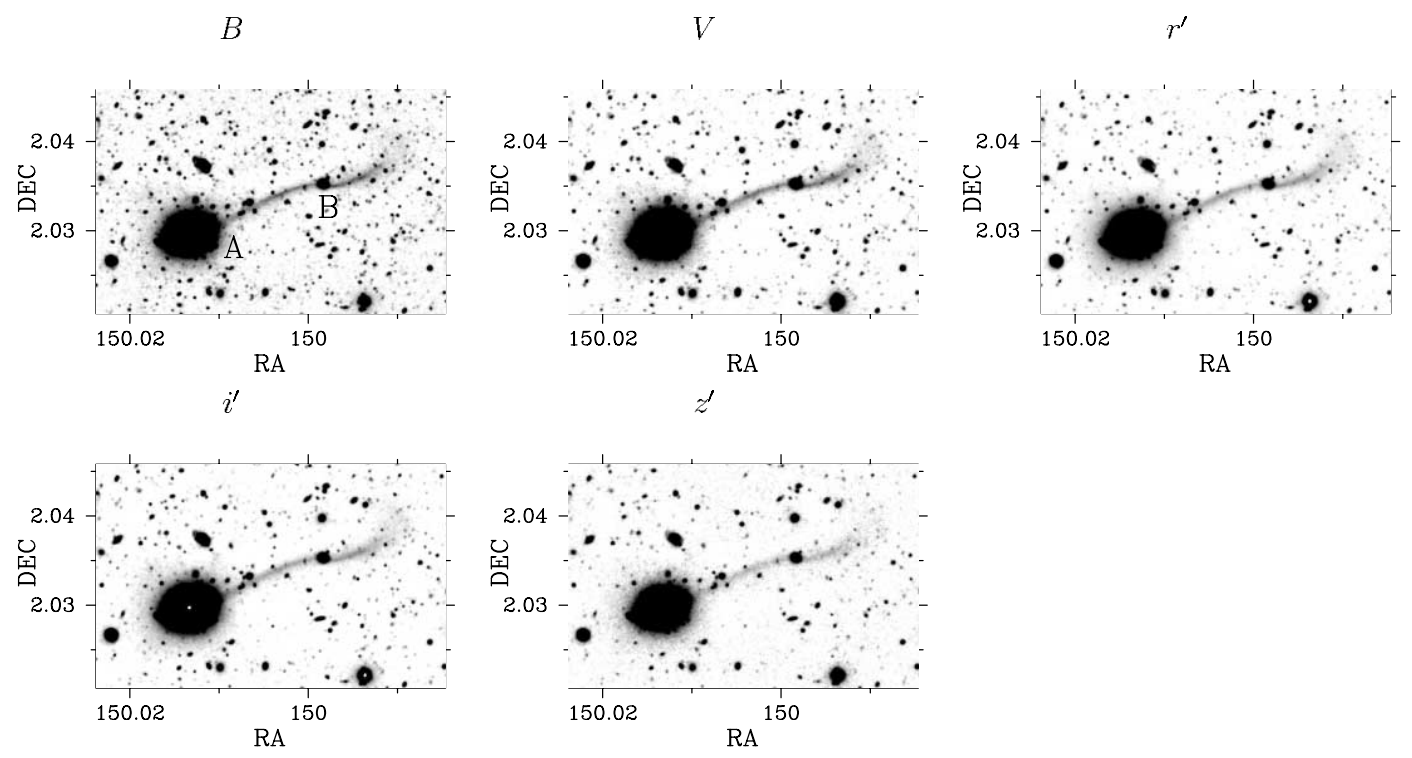

FIG. 1. - Suprime-Cam images of the threshing system. Image size of these figures are $2.3^{\prime} \times 1.5^{\prime}$ (corresponds to $243 \mathrm{kpc} \times 158 \mathrm{kpc}$ at $z \simeq 0.08$ ).

generally too faint to be detected in most cases. We present the photometric properties of the system and discuss some implications. We adopt a flat universe with $\Omega_{\text {matter }}=0.3, \Omega_{\Lambda}=0.7$, and $H_{0}=70 \mathrm{~km} \mathrm{~s}^{-1} \mathrm{Mpc}^{-1}$ throughout this paper, and we use the AB system for optical magnitudes.

The Cosmic Evolution Survey (COSMOS) is a treasury program on the Hubble Space Telescope (HST), awarded a total of $640 \mathrm{HST}$ orbits, to be carried out in two cycles (320 orbits in cycles 12 and 13 each; Scoville et al. 2006; Koekemoer et al. 2006). This is the largest amount of HST time ever allocated to a single project. COSMOS is a 2 square degree imaging survey of an equatorial field in $I(\mathrm{~F} 814 \mathrm{~W})$ band, using the Advanced Camera for Surveys (ACS).

\section{OBSERVATIONS}

The COSMOS HST survey alone cannot be used to address all scientific questions without additional ground-based and space-based follow-up observations at multiple wavelengths. We have been carrying out optical multiband imaging surveys of the COSMOS 2 square degree field centered at $\alpha(\mathrm{J} 2000.0)=$ $10^{\mathrm{h}} 00^{\mathrm{m}} 28.6^{\mathrm{s}}$ and $\delta(\mathrm{J} 2000.0)=+02^{\circ} 12^{\prime} 21.0^{\prime \prime}$ (Taniguchi et al.

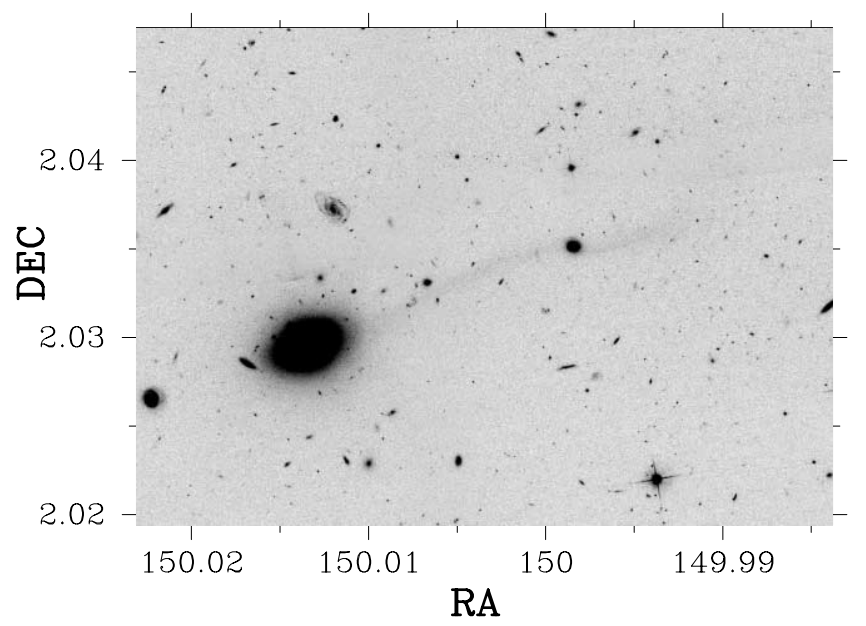

FIG. 2.-HSTACS $I-814$ band image of the galaxy threshing system. Image size of this figure is $2.3^{\prime} \times 1.5^{\prime}$.
2006; see also Capak et al. 2006) using Suprime-Cam, which consists of $5 \times 2$ CCDs with $2 \mathrm{k} \times 4 \mathrm{k}$ pixels and has a pixel scale of $0.202^{\prime \prime}$ pixel $^{-1}$ (Miyazaki et al. 2002) on the $8.2 \mathrm{~m}$ Subaru Telescope (Kaifu et al. 2000). During our two observing runs in 2004 January and February, we obtained $B-, V-, r^{\prime}-, i^{\prime}-$, and $z^{\prime}$-band images of the whole COSMOS field.

The individual CCD data were reduced and combined using our own data reduction software (Yagi et al. 2002) and IRAF. The combined images for individual bands were aligned and smoothed with Gaussian kernels to match the atmospheric seeing conditions. The FWHM of the PSF of the final images has been matched to $0.92^{\prime \prime}$, corresponding to the FWHM of the PSF in the $z^{\prime}$-band image that had the worst seeing of all the data. Photometric calibrations are made using spectrophotometric standard stars. Details of the data reduction processes for the COSMOS ACS data are given in Koekemoer et al. (2006).

\section{RESULTS}

In Figure 1, we show our five broadband images of the galaxy threshing system. The threshing system is a pair of interacting galaxies labeled $\mathrm{A}$ and $\mathrm{B}$ in the $B$-band image (Fig. 1). Galaxy A

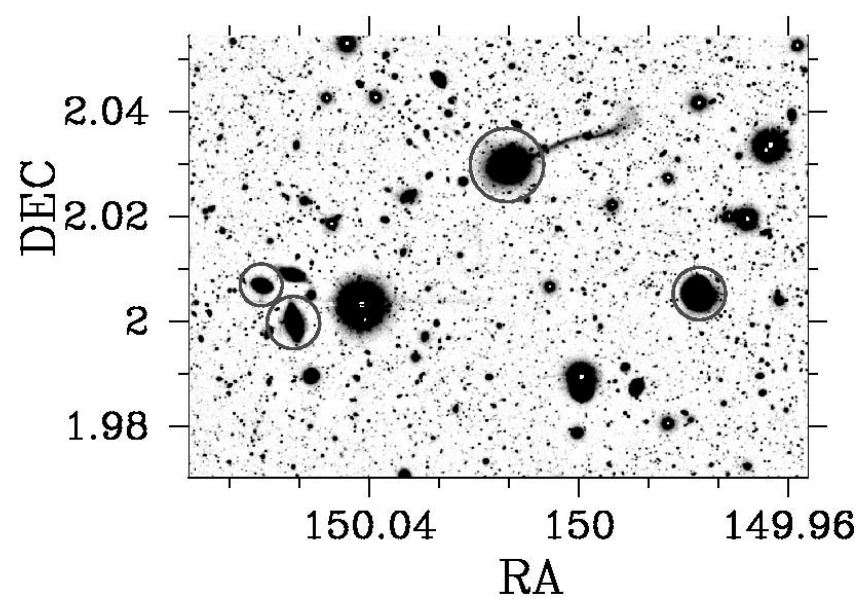

FIG. 3.-Circled galaxies are members of the galaxy group. They were identified by Merchán \& Zandivarez (2005). 


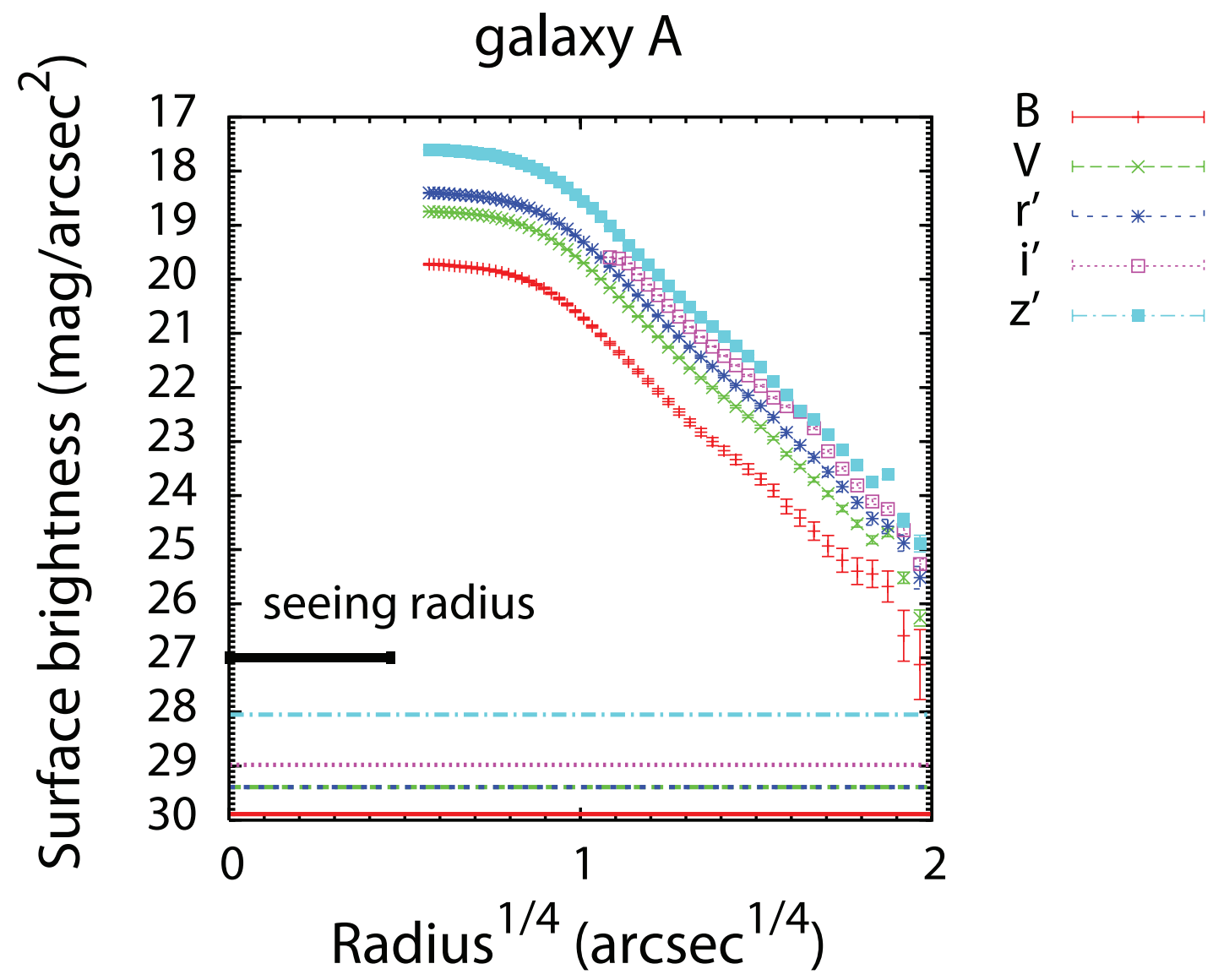

FIG. 4.- Surface brightness profiles of the threshing galaxy. Note that the central region $\left(<1^{\prime \prime}\right)$ of the threshing galaxy is saturated in $i^{\prime}$ and has been masked out. We also show the $1 \sigma$ of sky background and seeing radius $\left(=0.46^{\prime \prime}\right)$. The values of background are shown in Table 6 .

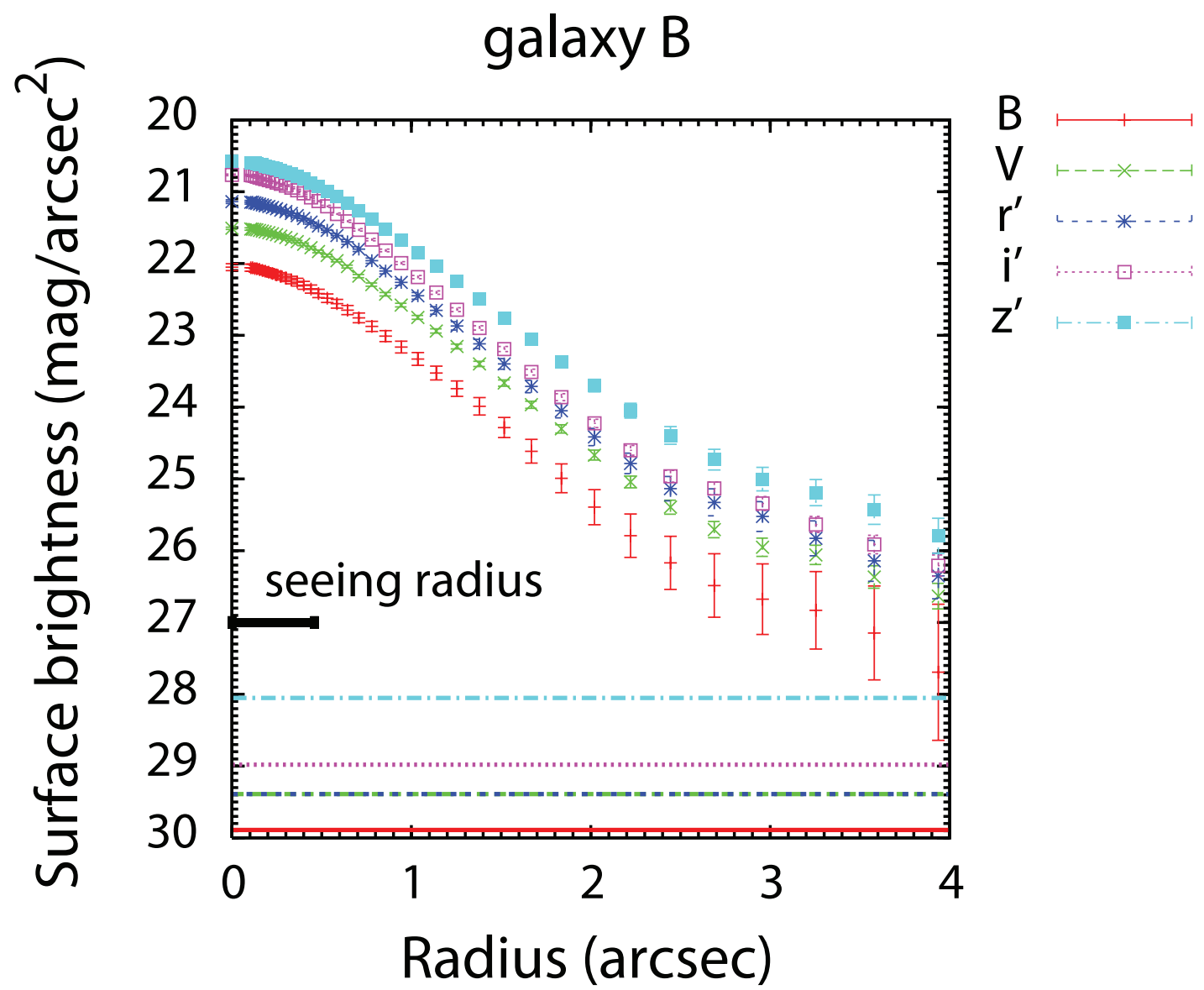

FIG. 5. - Surface brightness profiles of the threshed galaxy. We also show the $1 \sigma$ of sky background and seeing radius $\left(=0.46^{\prime \prime}\right)$. The values of background are shown in the Table 6 . 
TABLE 1

Basic Properties of the Threshing System

\begin{tabular}{|c|c|c|c|c|c|c|c|}
\hline Name & $\begin{array}{c}\text { R.A. } \\
(\mathrm{J} 2000.0)\end{array}$ & $\begin{array}{c}\text { Decl. } \\
(\mathrm{J} 2000.0)\end{array}$ & B & $V$ & $r^{\prime}$ & $i^{\prime}$ & $z^{\prime}$ \\
\hline J095959.6+020206(B) ..................... & 95959.61 & +20206.6 & $20.87 \pm 0.01$ & $20.15 \pm 0.01$ & $19.85 \pm 0.01$ & $19.56 \pm 0.01$ & $19.28 \pm 0.01$ \\
\hline
\end{tabular}

Notes._-Units of right ascension are hours, minutes, and seconds, and units of declination are degrees, arcminutes, and arcseconds. Magnitudes and their errors are derived using GALFIT.

appears to be a giant elliptical galaxy, while galaxy B appears to be a small satellite disk galaxy. It is remarkable that very long tidal tails are emanating from galaxy $\mathrm{B}$. These features are quite similar to those found in the first galaxy threshing system reported by Forbes et al. (2003). Although we have not yet obtained optical spectroscopy of galaxies A and B, their physical association appears to be real, and thus this system is the second case of galaxy threshing. In Figure 2, we show an ACS F814W image of this system. In the high-resolution HST image, there are many other satellite galaxies around galaxy A. This group has been identified as ID 10203 in SDSS by Merchán \& Zandivarez (2005), They are all large, bright galaxies $\left(r^{\prime} \sim 16-17\right)$ shown in Figure 3 . Note that galaxy B is not contained in the SDSS catalog, because the spectroscopic redshift of galaxy B was not obtained.

\subsection{Properties of the Two Galaxies in the Threshing System}

In order to study structural properties of the two galaxies in the threshing system, we examine their surface brightness distributions using the IRAF ellipse program. In Figure 4, we show the surface brightness profiles of galaxy $\mathrm{A}$ as a function of $r^{1 / 4}$. It follows a de Vaucouleur $r^{1 / 4}$ law, suggesting that it is an elliptical galaxy, consistent with the SDSS spectrum. In Figure 5, we show the surface brightness profile of galaxy $\mathrm{B}$ as a function of radius. It shows an exponential radial profile suggesting that this galaxy is probably a disk galaxy.

Next, we derive the apparent magnitudes of the two galaxies using GALFIT (Peng et al. 2002). We apply a de Vaucouleur $r^{1 / 4}$ model for galaxy A and an exponential disk model for galaxy B. Since the central region $\left(r<1^{\prime \prime}\right)$ of galaxy A is saturated in our $i^{\prime}$-band image, we have masked out this region. The magnitudes and their errors of both galaxies are summarized in Table 1. The scale length of galaxy $\mathrm{B}$ and the effective radius of galaxy A are shown in Table 2 and Table 3, respectively.

We also give results for the Sersic fitting in Table 4. The Sersic index of galaxy A ranges from $\approx 2.5$ to $\approx 3$, being smaller than 4 (which corresponds to a de Vaucouleur profile). Note that the central region of galaxy A is saturated in $i^{\prime}$, and thus the Sersic index may have large uncertainty. On the other hand, galaxy B has Sersic index around 1.4 in each filter. These results seem to be basically consistent with our interpretation that galaxy $\mathrm{A}$ is an elliptical galaxy, while galaxy $\mathrm{B}$ is a disk galaxy.

At a redshift of 0.08 (corresponding to a luminosity distance of $363.5 \mathrm{Mpc}$ ), we estimate an absolute $V$ magnitude (luminosity) of $M_{V} \sim-21.6\left(L_{V} \sim 3.7 \times 10^{10} L_{V, \odot}\right)$ for galaxy $\mathrm{A}$, and of $M_{V} \sim-17.7\left(L_{V} \sim 1.0 \times 10^{9} L_{V, \odot}\right)$ for galaxy B.

\subsection{Redshift of the Threshing System}

We found a spectroscopic redshift of $z=0.079$ for galaxy A (SDSS J100003.2+020146.4) in the SDSS spectroscopic data archive of third data release (Abazajian et al. 2005). Unfortunately, no spectroscopic information on the redshift is available for galaxy B. We derive a photometric redshift for galaxy B using the $\chi^{2}$ minimizing method (e.g., Lanzetta et al. 1996). We generate spectral energy distribution (SED) templates for $0 \leq z \leq 1.0$ with a redshift bin of $\Delta z=0.01$ using the population synthesis model GALAXEV (Bruzual \& Charlot 2003). The SEDs of local galaxies are well reproduced by models whose star formation rate (SFR) declines exponentially ( $\tau$ models); i.e., SFR $\propto \exp (-t / \tau)$, where $t$ is the age of the galaxy and $\tau$ is the timescale of star formation. We adopt $\tau=1$ Gyr models with a Salpeter initial mass function (a power index of $x=1.35$ and a stellar mass range of $\left.0.01 \leq m / M_{\odot} \leq 125\right)$ and solar metallicity, $Z=0.02$. We then calculate SEDs for ages of $t=1,2,3,4$, and $8 \mathrm{Gyr}$, corresponding to the SED of a starburst, Irr, Scd, Sbc, and elliptical galaxy, respectively. We adopt a visual extinction of $A_{V}=0$. Note that our threshing system consists of basically nearby galaxies. Therefore, our results should not be affected by metallicity and $A_{V}$. Applying this method, we obtain a photometric redshift of the galaxy B as $z_{\mathrm{ph}}=0.08$, being consistent with the spectroscopic redshift of the galaxy A. For the rest of the paper, we adopt $z=0.08$ for the galaxy threshing system $\left(1^{\prime \prime} \simeq 1.76 \mathrm{kpc}\right.$ at $\left.z=0.08\right)$.

\subsection{Properties of the Tidal Tails}

We investigate the morphological properties of the two tidal tails emanating from galaxy B. As shown in Figure 1, the two
TABLE 2

Scale Length of the Threshed Galaxy (GalaXy B, J095959.6+020206)

\begin{tabular}{ccc}
\hline Band & $\begin{array}{c}R_{S} \\
(\mathrm{arcsec})\end{array}$ & $\begin{array}{c}R_{S} \\
(\mathrm{kpc})\end{array}$ \\
\hline$B \ldots \ldots \ldots \ldots \ldots \ldots \ldots \ldots . .$. & 0.50 & 0.88 \\
& 0.58 & 1.02 \\
& 0.56 & 0.99 \\
$z^{\prime}$ & 0.53 & 0.93 \\
\hline
\end{tabular}

NotE.-These values are derived using GALFIT.
TABLE 3

Effective Radius of the Threshing Galaxy (GalaXy A, J100003.2+020146)

\begin{tabular}{ccc}
\hline \hline Band & $\begin{array}{c}R_{e} \\
(\mathrm{arcsec})\end{array}$ & $\begin{array}{c}R_{e} \\
(\mathrm{kpc})\end{array}$ \\
\hline$B \ldots \ldots \ldots \ldots . .$. & 3.29 & 5.97 \\
& 3.00 & 5.29 \\
& 3.20 & 5.64 \\
& 2.34 & 4.12 \\
\hline
\end{tabular}

NotE.-These values were derived using GALFIT. 
TABLE 4

Sersic Parameter of Both Galaxies

\begin{tabular}{|c|c|c|c|c|c|}
\hline Name & $B$ & $V$ & $r^{\prime}$ & $i^{\prime}$ & $z^{\prime}$ \\
\hline Galaxy A. & 2.91 & 2.56 & 2.69 & 1.80 & 2.57 \\
\hline 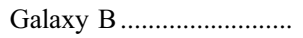 & 1.41 & 1.33 & 1.46 & 1.47 & 1.50 \\
\hline
\end{tabular}

Notes.- These values are derived using GALFIT. Note that the central region $\left(r<1\right.$ arcsec) of galaxy A is saturated in $i^{\prime}$.

long tidal tails are obvious in images of all bands, suggesting that they consist of stellar material. The lengths of the two tidal tails are $58^{\prime \prime}$ for the tail extending toward the galaxy A and $30^{\prime \prime}$ for the counter tail (east of galaxy B). These values correspond to lengths of $102 \mathrm{kpc}$ and $52 \mathrm{kpc}$, respectively in the plane of the sky.

The tail extending toward galaxy A appears to be narrower than the counter tail, whose width increases with increasing distance from galaxy B. This property is also seen in the first galaxy threshing system found by Forbes et al. (2003). In order to study this tendency more quantitatively, we examine the surface brightness distributions of the tails. In Figure 6, we show the $i^{\prime}$-band surface brightness profile perpendicular to the tails for the four spatial positions, a, b, c, and d, that are indicated in the right panel of Figure 6. As shown in the left panel of Figure 6 , the tail extending to galaxy $\mathrm{A}$ is significantly narrower than the counter one. The tail widths measured at $3 \sigma$ and $5 \sigma$ noise level for each position are given in Table 5 . The width at position $\mathrm{d}$ exceeds $10 \mathrm{kpc}$ even then it is measured at the $5 \sigma$ noise level. However, the width of the tail extending to galaxy A is only $\simeq 6-7 \mathrm{kpc}$.

There are some knotty structures in the tidal tails. It is likely that some of these may be tidal dwarf candidates (e.g., Zwicky 1956; Schweizer 1978; Barnes \& Hernquist 1992; Yoshida et al. 1994; Duc \& Mirabel 1998 and references therein). Future spectroscopic identification will be necessary to confirm them.
TABLE 5

Width of the Tidal Tails

\begin{tabular}{|c|c|c|c|c|}
\hline \multirow[b]{2}{*}{ TAIL } & \multicolumn{2}{|c|}{$3 \sigma$ WIDTH } & \multicolumn{2}{|c|}{$5 \sigma$ WIDTH } \\
\hline & $(\mathrm{kpc})$ & $(\operatorname{arcsec})$ & $(\mathrm{kpc})$ & $(\operatorname{arcsec})$ \\
\hline 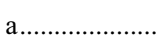 & $7.6_{-0.2}^{+0.1}$ & $4.3_{-0.1}^{+0.0}$ & $6.4 \pm 0.1$ & $3.6 \pm 0.0$ \\
\hline 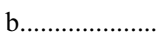 & $7.9_{-0.2}^{+0.3}$ & $4.5_{-0.1}^{+0.1}$ & $5.5_{-0.1}^{+0.2}$ & $3.1 \pm 0.1$ \\
\hline ............ & $11.8_{-1.2}^{+1.1}$ & $6.7 \pm 0.7$ & $8.5_{-0.4}^{+0.3}$ & $4.8_{-0.2}^{+0.1}$ \\
\hline d........................ & $12.3_{-0.2}^{+0.1}$ & $7.0 \pm 0.1$ & $10.3_{-0.5}^{+0.3}$ & $5.9_{-0.3}^{+0.2}$ \\
\hline
\end{tabular}

Note.-The positions of the cuts across the tidal tails are indicated in Fig. 6.

We estimate the mean surface brightness of the tidal tails in a sky area above $1.5 \sigma$ of the surface brightness in each band. The errors are estimated using the following relation:

$\sigma^{2}($ mean counts $/$ pixel $)=\frac{(\text { mean counts } / \text { pixel })+\sigma_{\text {background }}^{2}}{N}$.

$N$ is the total number of pixels of tidal tail, and $\sigma_{\text {background }}^{2}$ is $1 \sigma$ sky background noise. The results are given in Table 6 . The mean surface brightness is fainter by $\approx 1$ magnitude than that of the tail found by Forbes et al. (2003).

We also estimate the total magnitude of the tidal tails in each band. Errors are estimated using the following relation:

$$
\sigma^{2}(\text { total counts })=\text { total counts }+N \sigma_{\text {background }}^{2} .
$$

The results are given in Table 6. It is found that the magnitude of the tails is comparable to that of galaxy B in each band.

In Figure 7, we show the $B-r^{\prime}$ color distribution in the galaxy threshing system. The mean $B-r^{\prime}$ color of galaxy A is $1.37 \pm$ 0.06 , while, that of galaxy B is $1.02 \pm 0.07$. There is a small color difference between the main body of galaxy B and the two tidal tails. This suggests that stars located in the tidal tails were dispersed from the main body of galaxy $\mathrm{B}$, providing strong evidence
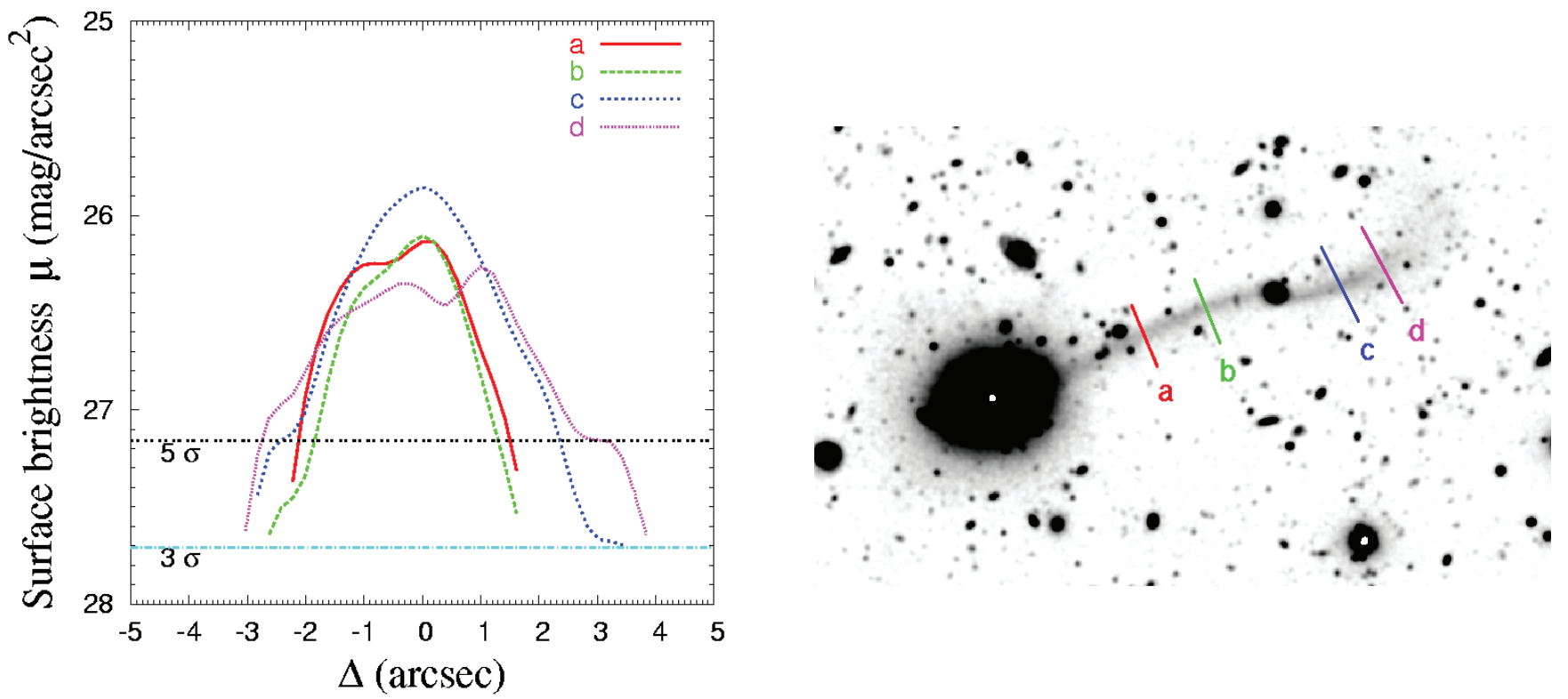

FIG. 6.- Surface brightness distribution perpendicular to the extent of the tidal tails as a function of position along the tails. The investigated spatial positions are marked by bars in the right panel. The surface brightness profile at each position is shown in the left panel. 
TABLE 6

Properties of the Tidal Tails

\begin{tabular}{|c|c|c|c|c|c|}
\hline Parameter & $B$ & $V$ & $r^{\prime}$ & $i^{\prime}$ & $z^{\prime}$ \\
\hline Mean Surface Brightness (mag $\left.\operatorname{arcsec}^{-2}\right) \ldots \ldots \ldots \ldots$ & $27.90 \pm 0.08$ & $27.30 \pm 0.04$ & $27.11 \pm 0.07$ & $26.84 \pm 0.04$ & $26.29 \pm 0.05$ \\
\hline 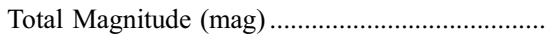 & $20.91 \pm 0.07$ & $20.18 \pm 0.05$ & $19.82 \pm 0.05$ & $19.66 \pm 0.04$ & $19.23 \pm 0.05$ \\
\hline 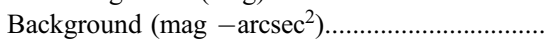 & 29.89 & 29.39 & 29.39 & 28.98 & 28.05 \\
\hline
\end{tabular}

that galaxy threshing has been occurring in this system. The total magnitude of the tidal tails is comparable to that of the main body of galaxy B. We estimated that the luminosity of galaxy B is $L_{V} \sim\left(1.03 \pm 9.48 \times 10^{-5}\right) \times 10^{9} L_{V, \odot}$, and that of the tail is $L_{V} \sim$ $\left(0.94 \pm 2.16 \times 10^{-3}\right) \times 10^{9} L_{V} . \odot$. These luminosities are nearly the same. This suggests that galaxy B lost about half of its former mass during the (ongoing) threshing event.

\subsection{Dynamical Properties of the Galaxy Threshing System}

We assume that galaxy A has a mass-to-light ratio of 10 typical for an elliptical galaxies and 3 for galaxy $\mathrm{B}$. Given the $V$-band luminosity in $\S 3.1$, we obtain $M_{\mathrm{A}} \simeq 3.7 \times 10^{12} \mathcal{M}_{\odot}$ and $M_{\mathrm{B}} \simeq$ $3.1 \times 10^{9} \mathcal{M}_{\odot}$ for $\mathrm{A}$ and $\mathrm{B}$, respectively.

Next, we estimate the probable pericenter distance $\left(r_{p}\right)$ for the galaxy threshing event. As shown in Figure 5, galaxy B shows an exponential surface brightness profile for $r \lesssim 2.9^{\prime \prime}$. If we assume that the mass outside of this radius has been expelled during the previous encounter between the two galaxies, and we use the equation of tidal radius as a rough guide of the pericenter distance, we can then estimate the pericenter as follows:

$$
r_{p} \simeq r\left(\frac{2 \mathcal{M}}{m}\right)^{1 / 3}
$$

Using the derived masses of the two galaxies, we obtain a pericenter distance of $r_{p} \simeq 68 \mathrm{kpc}$. Bekki et al. (2003) made general numerical simulations of a galaxy threshing systems with $r_{p}=$ $65 \mathrm{kpc}$. Mayer et al. (2001) used a similar value, $r_{p}=75 \mathrm{kpc}$. Our estimate is consistent with the results of these numerical simulations.

The projected separation between two galaxies is $102 \mathrm{kpc}$. If we adopt an average relative velocity between the two galaxies of $200 \mathrm{~km} \mathrm{~s}^{-1}$, we find that the previous encounter occurred $\tau \sim 5 \times 10^{8}\left(v / 200 \mathrm{~km} \mathrm{~s}^{-1}\right)^{-1}$ yr ago.

Galaxy B found in this study will merge into galaxy A after a few billion years after (see Bekki et al. 2001). Even in a facial phase of such minor mergers, faint remnants could be found around the host galaxy.

Recently, morphological evidence for such a final phase of minor mergers has been found around field red elliptical galaxies (van Dokkum 2005). Deep optical imaging survey for ordinarylooking galaxies should provide more information on the global, and dynamical evolution of galaxies.

\section{CONCLUDING REMARKS}

In this paper, we have presented the discovery of a new potential galaxy threshing system. Although merging systems and

$$
B-r^{\prime}
$$
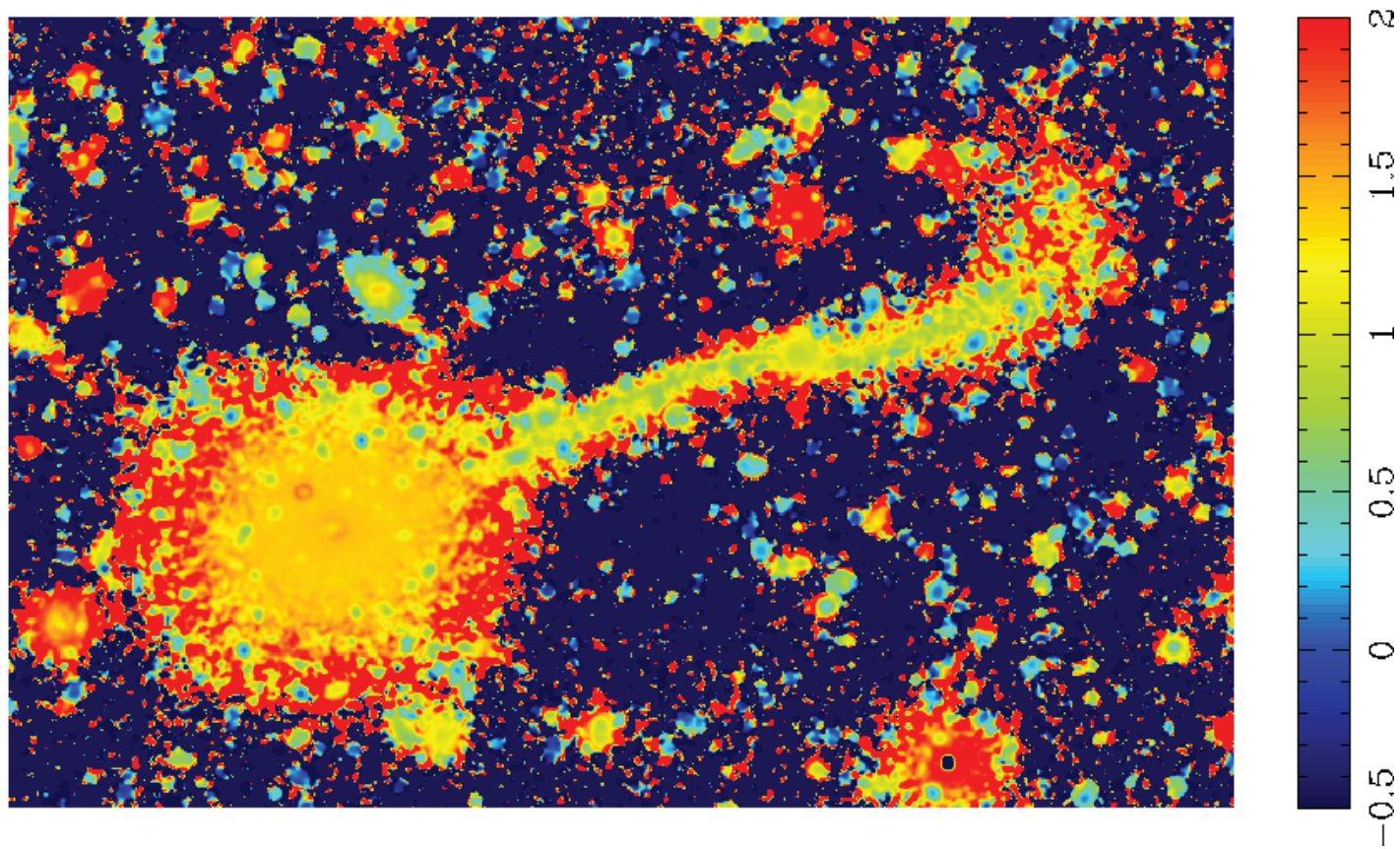

FIG. 7. $-B-r^{\prime}$ color map. Image size of this figure is $2.3^{\prime} \times 1.5^{\prime}$. Outer parts of the threshing system (in particular, galaxy A) show redder colors. However, this feature is due to poor signal-to-noise ratio because of much lower surface brightness. 
interacting systems are often observed, it is rare to find faint tidal tails in ongoing minor mergers because their tidal tails are generally too faint to be detected. Our discovery is important because we find clear evidence for the disruption of a small satellite galaxy. This is only the second well-defined galaxy threshing system found so far.

The HST COSMOS Treasury program was supported through NASA grant HST-GO-09822. We wish to thank Tony Roman, Denise Taylor, and David Soderblom for their assistance in planning and scheduling of the extensive COSMOS observations. We gratefully acknowledge the contributions of the entire COSMOS collaboration consisting of more than 70 scientists. More information on the COSMOS survey is available at http:// www.astro.caltech.edu/ cosmos. It is a pleasure the acknowl- edge the excellent services provided by the NASA IPAC/IRSA staff (Anastasia Laity, Anastasia Alexov, Bruce Berriman, and John Good) in providing on-line archive and server capabilities for the COSMOS data sets. The COSMOS Science meeting in 2005 May was supported in part by the NSF through grant OISE0456439. We would like to thank the Subaru Telescope staff for their invaluable assistance. IRAF (Image Reduction and Analysis Facility) is distributed by the National Optical Astronomy Observatory, which is operated by the Association of Universities for Research in Astronomy, Inc., under cooperative agreement with the National Science Foundation. This work was financially supported in part by the Ministry of Education, Culture, Sports, Science, and Technology (Nos. 10044052 and 10304013), and by the Japan Society for the Promotion of Science (15340059 and 17253001). S. S. S. and T. N. are financially supported by the Japan Society for the Promotion of Science (JSPS) through JSPS Research Fellowship for Young Scientists.
Abazajian, K., et al. 2005, AJ, 129, 1755

Arp, H. 1966, ApJS, 14, 1

Barnes, S. A., \& Hernquist, L. 1992, Nature, 360, 715

Bekki, K., Couch, W. J., \& Drinkwater, M. J. 2001, ApJ, 552, L105

Bekki, K., Couch, W. J., Drinkwater, M. J., \& Shioya, Y. 2003, MNRAS, 344, 399

Bruzual, G., \& Charlot, S. 2003, MNRAS, 344, 1000

Capak P., et al. 2007, ApJS, 172, 284

de Grijs, R., Lee, J. T., Mora Herrera, M. C., Fritze-v. Alvensleben, U., \& Anders, P. 2003, NewA, 8, 155

Drinkwater, M. J., Jones, J. B., Gregg, M. D., \& Phillipps, S. 2000a, PASA, 17, 227

Drinkwater, M. J., et al. 2000b, A\&A, 355, 900

Duc, P. A., \& Mirabel, I. F. 1998, A\&A, 333, 813

Ellis, R., Santos, M. R., Kneib, J.-P., \& Kuijken, K. 2001, ApJ, 560, L119

Forbes, D. A., Beasley, M. A., Bekki, K., Brodie, J. P., \& Strader, J. 2003, Science, 301, 1217

Kaifu, N., et al. 2000, PASJ, 52, 1

Kneib, J. -P., Ellis, R. S., Santos, M. R., \& Richard, J. 2004, ApJ, 607, 697

Koekemoer, A., et al. 2007, ApJS, 172, 196

Lanzetta, K. M., Yahil, A., \& Fernández-Soto, A. 1996, Nature, 381, 759

Mayer, L., Governato, F., Colpi, M., Moore, B., Quinn, T., Wadsley, J., Stadel, J., \& Lake, G. 2001, ApJ, 559, 754

Merchán E. M., \& Zandivarez, A. 2005, ApJ, 630, 759

Miyazaki, S., et al. 2002, PASJ, 54, 833

\section{REFERENCES}

Ostriker, J. P., \& Tremaine, S. 1975, ApJ, 202, L113

Pascarelle, S. M., Windhorst, R. A., \& Keel, W. C. 1998, AJ, 116, 2659

Peebles, P. J. E. 1993, Principles of Physical Cosmology (Princeton: Princeton Univ. Press)

Peng, Y. C., et al. 2002, AJ, 124, 266

Santos, M. R., Ellis, R. S., Kneib, J. -P., Richard, J., \& Kuijken, K. 2004, ApJ, 606,683

Schweizer, F. 1978, in Structure and Properties of Nearby Galaxies, ed. E. M. Berkhuijsen \& R. Wieleinski (Dordrecht: Reidel), 279

Scoville, N. Z., et al. 2007, ApJS, 172, 1

Taniguchi, Y., Shioya, Y., Fujita, S. S., Nagao, T., Murayama, T., \& Ajiki, M. 2003a, JKAS, 36, 123 (erratum 36, 283)

Taniguchi, Y., et al. 2003b, ApJ, 585, L97 2007, ApJS, 172, 9

Toomre, A., \& Toomre J. 1972, ApJ, 178, 623

Tran, H., et al. 2003, ApJ, 585, 750

Tremaine, S. 1981, in The Structure and Evolution of Normal Galaxies, ed. S. M. Fall \& D. Lynden-Bell (Cambridge: Cambridge Univ. Press), 67 van Dokkum, P. G. 2005, AJ, 130, 2647

Yagi M., Kashikawa, N., Sekiguchi, M., Doi, M., Yasuda, N., Shimasaku, K., \& Okamura, S. 2002, AJ, 123, 66

Yoshida, M., Taniguchi, Y., \& Murayama, T. 1994, PASJ, 46, L195

Zaritsky, D., Smith, R., Frenk, C., \& White, S. D. M. 1997, ApJ, 478, 39

Zwicky, F. 1956, Multiple Galaxies, Ergenisse Exakten Naturwissenschaften, 29,344 